\title{
COVID-19 Pandemic: Evaluation of Socio-Economic Impact on Aesthetic Plastic Surgery Providers
}

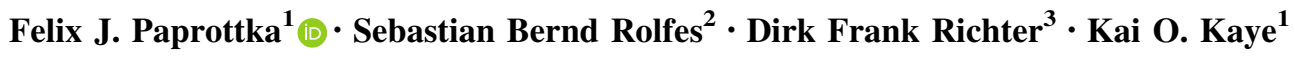

Received: 19 October 2020/Accepted: 4 January 2021/Published online: 8 April 2021

(C) Springer Science+Business Media, LLC, part of Springer Nature and International Society of Aesthetic Plastic Surgery 2021

\begin{abstract}
Background In many countries, the worldwide spread of COVID-19 has led to a near total stop of non-urgent, elective surgeries across all specialties during the first wave's peak of the pandemic. For providers of aesthetic surgery procedures or minimal invasive cosmetic treatments, this led to a huge socio-economic impact worldwide. In order to evaluate valid clinical management strategies for future pandemic events and to overcome the challenges imposed by the current pandemic, it is paramount to analyse the socio-economic effects caused by the COVID-19 crisis.

Methods An online survey comprising 18 questions was sent out five times by e-mail to all members of the International Society of Aesthetic Plastic Surgery (ISAPS) between June and August 2020. The data set was statistically analyzed and grouped into an overall group and into subgroups of countries with high $(n=251)$ vs. low $(n=440)$ gross domestic product per capita (GDP p.c.) and five defined world regions (Europe $(n=214)$; North
\end{abstract}

Supplementary Information The online version of this article (https://doi.org/10.1007/s00266-021-02130-9) contains supplementary material, which is available to authorized users.

Felix J. Paprottka

dr.paprottka@oceanclinic.net

1 Center for Plastic and Aesthetic Surgery, Ocean Clinic, Av. Ramón y Cajal 7, 29601 Marbella, Málaga, Spain

2 Associate Lecturer of Empirical Communications Research and Statistics, International Media Systems Research and Media and Information Governance, zeb business school / Steinbeis University, Münster \& Berlin, Germany

3 Department for Plastic Surgery, Dreifaltigkeitskrankenhaus Wesseling, Wesseling, Germany
America (NA; $\mathrm{n}=97$ ); South America (SA; $n=206$ ); Asia and Oceania (Asia + OC; $n=99$ ); Africa and Middle East $($ Africa + ME; $\mathrm{n}=75)$ ).

Results A total of 691 recipients completed the survey. The majority of the participants experienced severe operating restrictions resulting in a major drop of income from surgical patients. Low GDP p.c. countries experienced a bigger negative economic impact with less aesthetic (non-) surgical procedures, whereas the high GDP p.c. subgroup was less affected by the COVID-19 crisis. Most of the survey participants had already adopted the ISAPS guidelines for patient (pre-) appointment screening and clinical/patient-flow management. For surgical and nonsurgical aesthetic procedures, in the high GDP p.c. subgroup more basic-level PPE (surgical mask) was used, whereas the low GDP p.c. subgroup relied more on advanced-level PPE (N-95 respirator mask or higher). Comparing the different world regions, Europe and Africa used more basic-level PPE.

Conclusions Measurable differences in the socio-economic impact and in the adaptation of safety protocols between high and low GDP p.c. subgroups and between different world regions were present. Since the COVID-19 pandemic is an international crisis, aligned, expedient and universal actions should be taken.

Level of evidence $V$ This journal requires that authors assign a level of evidence to each article. For a full description of these Evidence-Based Medicine Ratings, please refer to Table of Contents or online Instructions to Authors www.springer.com/00266.

Keywords COVID-19 - SARS-CoV-2 - Pandemic · Plastic surgery $\cdot$ Cosmetic surgery $\cdot$ Aesthetic surgery $\cdot$ Botulinum toxin (botox) - Filler - Survey - Private clinic · Private practice · ork conditions - ISAPS · Guidelines · 
International · Clinical management $\cdot$ Hygiene $\cdot$ Personal protective equipment (PPE) - Patient traffic management . Surgical and aestheticnon-surgical work-flow - Daily clinic workflow $\cdot$ Pre-appointment screening

\section{Introduction}

The novel COVID-19 disease has become a major threat to the worlds' health systems and their economies [1]. At the moment, a rapid spread of SARS-CoV-2 can be observed, for example, in the USA, Brazil, India, France, Spain, South Africa, Russia and various other countries in spite of all previous infection control measures [2,3]. Germany and Spain actually have to manage numerous new COVID-19 hotspots, mostly in urban areas with low socio-economic background, or in production areas with working conditions that make social distancing difficult. This has already led to a new lockdown of the Spanish capital Madrid and a partial lockdown in the rest of the country $[3,4]$.

With most European countries approaching the peak of the second pandemic wave, the reevaluation of safety and infection management protocols regains importance. Especially in countries that experienced mayor restrictions on social life or had a complete lockdown during the first wave, lifting of these restrictions led to a bounce-back effect. Despite new achievements in vaccination research, a vaccine may not be available globally for the general public before mid of 2021 [1]. Therefore, the current pandemic poses a major threat to all medical specialties, especially to those mostly performing elective surgeries. This creates a need to define safety protocols that help hedging the pandemic risk. Recently, the International Society of Aesthetic Plastic Surgery (ISAPS) published their COVID-19 guidelines and safety protocols for aesthetic surgery providers based on a publication by Kaye et al. (2020) [1]. The guidelines include recommendations on adaptions of the preanesthetic/anaesthesia circuit, on COVID-19 testing for elective surgery, the use of personal protective equipment (PPE) and workflow recommendations for consultations, patient selection, clinical admission and administration in general.

A higher mortality in COVID-19+ patients undergoing surgery has been demonstrated [5]. Also an increased rate of peri- and post-operative complications such as pulmonary dysfunction, thrombosis, fever or a more severe evolution of the disease in the post-operative phase has been reported after an SARS-CoV-2 infection [1, 5]. Due to a long incubation time ranging from several days up to 2 weeks, asymptomatic carriers undergoing elective aesthetic surgery are a potential risk [1]. A high infection risk for healthcare workers during the induction and termination of intubation anaesthesia (ITA) in COVID-19+ patients has been observed ${ }^{6}$, while asymptomatic patients undergoing ITA might experience a more severe evolution of the disease through ventilation ${ }^{1}$. The immune system alteration associated with mayor surgical procedures presents additional risks for the COVID-19+ patients undergoing elective surgery. [1]

In general, an augmentation in age, pre-morbidities and operation time correlates with a higher risk for perioperative complications and a higher mortality rate in COVID$19+$ patients [1]. These augmented risks impose a challenge for all elective surgery providers, and especially the aesthetic surgery community has to implement solid strategies to cope with the challenge and to mitigate associated risks. To evaluate these coping strategies and to compare the implementation of safety protocols on an international level, the authors created an online survey to assess the current situation of private practices and clinics within the field of aesthetic surgery and aesthetic medicine.

\section{Material and Method}

\section{Data Collection}

An online survey comprised of 18 questions evaluating the strategies and protocols used during the COVID-19 pandemic (e.g. use of PPE, patient traffic management, surgical and aesthetic non-surgical (non-invasive) patient flow and patient screening) was designed and a sent out to all recipients of the ISAPS general mailing list $(n=24426$; each recipient could only participate once in the survey; time interval to complete survey: June-August 2020). A reminder was sent out after 2, 4, 6 and 8 weeks. For each question, only one answer could be selected.

The ISAPS guidelines referred to in this article are based on a large literature meta-analysis by Kaye et al. ${ }^{1}$ and include recommendations for clinical management, risk stratification, patient testing, and level of PPE usage for elective surgery during the COVID-19 pandemic (documents are available for download on the ISAPS website).

The location data of each survey participant were used to divide countries by high and low gross domestic product per capita (GDP p.c.). The "high GDP p.c." group $(n=251)$ includes only the TOP30 countries with the highest GDP p.c. while the "low GDP p.c." group ( $n=440)$ encompasses all the remaining countries with a lower GDP p.c.. The state ranking was based on published data of the World Bank [7]. Table 1 shows the submissions from high GDP p.c. countries.

For definition of statistical sample size, the survey participants of the "high GDP p.c." group $(251 / 691$; 36\%) 
Table 1 High GDP countries (TOP30). Listing of the 30 highest GDP p.c. countries $(n=251)$ in descending order. The ranking was based on the official data of the World Bank [7], which can be retrieved from the institution's web page.

\begin{tabular}{|c|c|c|}
\hline $\begin{array}{l}\text { HIgh GDP } \\
\text { Ranking (Top 30) }\end{array}$ & Country & $\begin{array}{l}\text { Survey } \\
\text { participants }(n)\end{array}$ \\
\hline 1 st & Monaco & 0 \\
\hline 2nd & Liechtenstein & 0 \\
\hline $3 \mathrm{rd}$ & Luxembourg & 0 \\
\hline 4th & Switzerland & 8 \\
\hline 5th & Ireland & 2 \\
\hline 6th & Norway & 2 \\
\hline 7 th & Iceland & 0 \\
\hline 8 th & USA & 89 \\
\hline 9 th & Singapore & 3 \\
\hline 10th & Qatar & 1 \\
\hline 11th & Denmark & 3 \\
\hline 12 th & Australia & 15 \\
\hline 13th & Netherlands & 6 \\
\hline 14th & Sweden & 1 \\
\hline 15 th & Austria & 3 \\
\hline 16 th & Finland & 2 \\
\hline 17 th & San Marino & 0 \\
\hline 18th & Germany & 24 \\
\hline 19th & Canada & 11 \\
\hline 20th & Belgium & 11 \\
\hline $21 \mathrm{st}$ & Israel & 3 \\
\hline 22nd & United Arab Emirates & 5 \\
\hline $23 \mathrm{rd}$ & United Kingdom & 25 \\
\hline 24th & New Zealand & 2 \\
\hline 25th & Andorra & 0 \\
\hline 26th & France & 11 \\
\hline 27th & Japan & 5 \\
\hline 28th & Italy & 17 \\
\hline 29th & Bahamas & 1 \\
\hline 30th & Kuwait & 1 \\
\hline
\end{tabular}

were set as one third while the "low GDP p.c." group (440/ $691 ; 64 \%$ ) was predefined as two-thirds of all survey participants.

For further analysis, the data set was also divided into five world regions (Europe $(n=214)$; North America (NA; $n=97)$; South America (SA; $n=206$ ); Asia and Oceania (OC) $($ Asia + OC; $n=99)$; Africa and Middle East (ME) (Africa + ME; $n=75$ ).

Russia was defined to be an Asian country. The ME is formed by Bahrain, Cyprus, Egypt, Iran, Iraq, Israel, Jordan, Kuwait, Lebanon, Oman, Qatar, Saudi Arabia, Syria,
Turkey, United Arab Emirates and Yemen. Hongkong was counted as being part of China. Oceania (OC) consists of the following countries: Australia, Brunei, Cook Islands, East Timor, Fiji, Indonesia, Kiribati, Malaysia, Marshall Islands, Micronesia, Nauru, New Zealand, Niue, Palau, Papua New Guinea, Philippines, Samoa, Solomon Islands, Tonga, Tuvalu and Vanuatu.

Advanced-level PPE was defined as using N-95, KN-95 or FFP2/3 respirator masks, in combination with eye protection and/or double gloving_having a protecting ability for the user against SARS-CoV-2. Basic-level PPE was defined as using only surgical mask and single gloving.

\section{Statistical Analysis}

A total of 18 questions (Q) with discrete, mostly nominal variables, each with 4 or 5 attributes encoded by answer numbers $\left(n_{\mathrm{j}}\right)$, were tested in a random sample of $n=(673$; 691) of actual responses per question Q1-18 comparing two GDP-related group samples and five world regions $\left(n_{\mathrm{i}}\right)$. The survey's attributes were not consistently powerful, unambiguous or bijective in a one-to-one correspondence as they overlapped in categories, with variables on an ordinal and interval scale. To compensate these differing scales and to take in account small group sample sizes for $\mathrm{n}_{\mathrm{i}}$, differences between answer distributions of $n_{i j}$ were tested with a Chi-square test $\left(\chi^{2}\right)$ of homogeneity between group samples and a Chi-square test of independence [8] for each groups' answer distribution from the global distribution (= overall group). Chisquare test of homogeneity between group samples and Chi-square test for independence from the overall answer distribution were performed at $\alpha=0.05$. In all answer distributions, where group sample size and test method fulfilled the asymptotic condition for expected values $\left(\mathrm{n}_{\exp }\right.$ $>5$ ) and confirmed a Chi-square distribution, significance testing was performed on all variables' scales of measurement. In answer distributions with insufficient sample size for precise significance testing of $\mathrm{H}_{\mathrm{o}}$ on a Chi-square distribution, affected variables were isolated. For world regions group samples Q2-3, Q6 and Q9-12 and for GDPrelated group samples Q1-4, Q6-12 and Q16-18, the answer distributions were Chi-square distributed. Significance, when mentioned in this publication, refers to the global overall distribution ( $=$ in comparison with overall group). A Spearman rank correlation test ${ }^{9}$ was conducted to assess the global overall answer distribution and statistically dependence based on relative answer frequency distributions. Data collection and the primary statistical analysis were performed with Microsoft Excel $^{\circledR}$ (Microsoft Corporation, Redmond, USA). 


\section{Results}

A total of 691 mailing list subscribers completed the survey $(n=691 ; 2,8 \%)$. The answers to all 18 questions were analyzed for different outcomes in high and low GDP p.c. (Figs. 1a-9a) and for different regions of the participants' origin (Europe; NA; SA; Asia + OC; Africa + ME; Figs. 1b-9b). The answers with a significant statistical power in relation to the overall group are marked with $*$ in Figs. 1A/B-9A/B and the corresponding $r_{\mathrm{s}}$ values are listed in the respecting figure legends.

Comparing the distribution of region of origin of the survey participants with the ISAPS membership statistic (status: 08/2020), each regional group sample reflects the official ISAPS members distribution by world region with a deviation of no more than 5\% (Europe (ISAPS: 31\%; our data: $31 \%(+/-0 \%)$ ); NA (ISAPS: $10 \%$; our data: $14 \%$ $(+4 \%)$ ); SA (ISAPS: $27 \%$; our data: $30 \%(+3 \%)$; Asia + OC (ISAPS: $19 \%$; our data: $14 \%(-5 \%)$ ); Africa + ME (ISAPS: $13 \%$; our data: $11 \%(-2 \%)$ ).

\section{Discussion}

In the authors' opinion, the data presented in this article allow a solid assessment of the impact of the current COVID-19 pandemic on aesthetic surgery service providers worldwide, and up to now, it is the largest known international collection about the socio-economic impact on this topic. Despite the high absolute number of participants, that completed our survey $(n=691)$, only a low response rate of $2.8 \%$ was given. Nevertheless, our data set resembles the official statistics about ISAPS membership in relation to the five world regions (with only minor deviations), and thus, the authors of this publication believe in the accuracy and the usefulness of the presented data. Our data set represents a reliable and unique source of information for all plastic surgeons around the world and helps to understand the socio-economic effects behind the COVID-19 pandemic.

One limitation of the survey is that not all countries are represented. Also countries with a very high level of survey participation might bias the outcomes of their respective world regions (e.g. Mexico's impact $(n=63)$ on region South America; Greece's impact $(n=21)$ on region Europe). Additionally, countries with a low density of aesthetic service providers but a high survey participation rate might be overrepresented, whereas countries with a high density of aesthetic service providers but a low survey participation rate might be underrepresented.

Despite these weakness, which cannot be changed anymore because (snapshot in time), the authors strongly believe that this publication will be of value for many readers.

\section{Question 1-Work Disturbance Due to the Pandemic (Fig. $1 \mathrm{~A} / \mathrm{B}$ )}

The majority of survey participants experienced prolonged operating restrictions (overall group: 56\% (> 8weeks); $29 \%$ (4-7 weeks)) with no significant differences in duration between countries with high GDP p.c. vs. countries with low GDP p.c. (Fig. 1a). In the ranking of the different world regions, survey participants from NA experienced the longest disruptions with more than 8 weeks (Fig. 1b). This is most probably due to the strong impact of the COVID-19 pandemic in the USA, as US survey participants represent $89 \%$ of the NA group.

\section{Question 2-3-(Non-) Surgical Patient Traffic (Fig. 2-3 A/B)}

Eighty-one per cent of the low GDP p.c. countries reported a significantly reduction ( $25 \%$ or more) in demand for surgical and non-surgical procedures after resuming activity, while 49 $\%$ of the high GDP p.c. countries reported no significant changes or even more demand (Figs. 2a/3a). Comparing the NA and SA subgroups to the overall group, both groups showed a significantly higher reduction in demand, while Europe, Asia $+\mathrm{OC}$ and Africa $+\mathrm{ME}$ seem to have been less affected (Fig. 2b/3b). Since our survey was conducted during summer of 2020, it is also not surprising that the USA showed less patient traffic at that time, because they got hit hard by the SARS-CoV-2 virus pandemic especially during these months. Additionally, these data confirm that the COVID-19 pandemic already had a measurable economic impact on aesthetic surgery providers worldwide, which is likely to worsen with any additional pandemic wave. Nevertheless, there were as well some countries, which had in general a strong demand for plastic surgery procedures [12]. But these countries were mainly part of the high GDP p.c. group (except South Korea) [12]. Furthermore, the authors believe that not a loss of patient interest in aesthetic treatments was the reason for the drop of cosmetic (non-)surgical procedures in the effected countriesbut also travel restrictions, shortages of advanced-level PPE and/or partial lockdowns, which might have had a stronger effect on private clinics, need to be mentioned.

\section{Question 4-ISAPS Recommendations for (Pre-) Appointment Screening (See Online Material; Fig. I A/B)}

Eighty-seven per cent of all participants reported implementation of three or more of the five measures recommended in the ISAPS guidelines for (pre-) appointment 
$1 \mathrm{~A}$

If you stopped practicing, how many weeks after pandemic peek in YOUR country/region did YOU resume elective aesthetic surgery procedures?

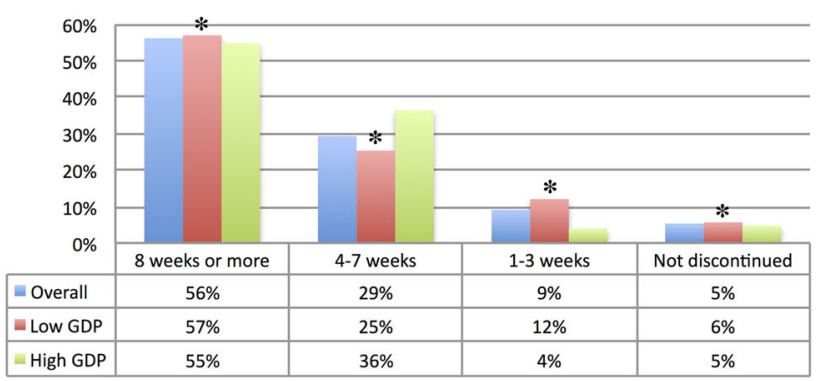

3A

How would YOU estimate patient traffic and demand for aesthetic non-surgical procedures since resuming activity?

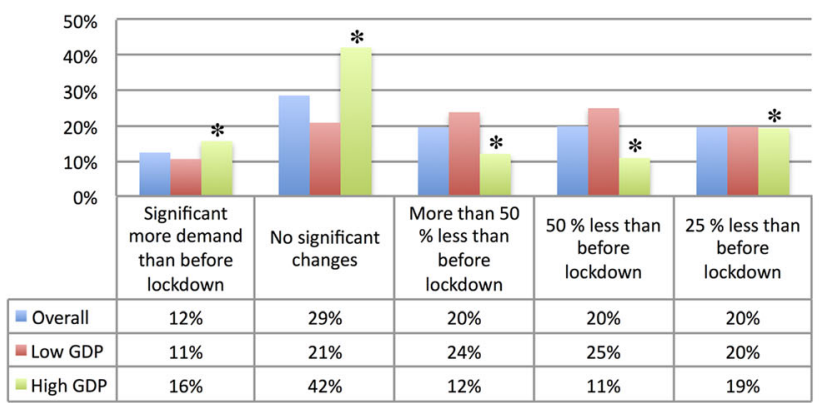

5A

How high is the percentage of positive tests ( $P C R$ or rapid $A B$ ) within YOUR patient cohort since resuming elective activity?

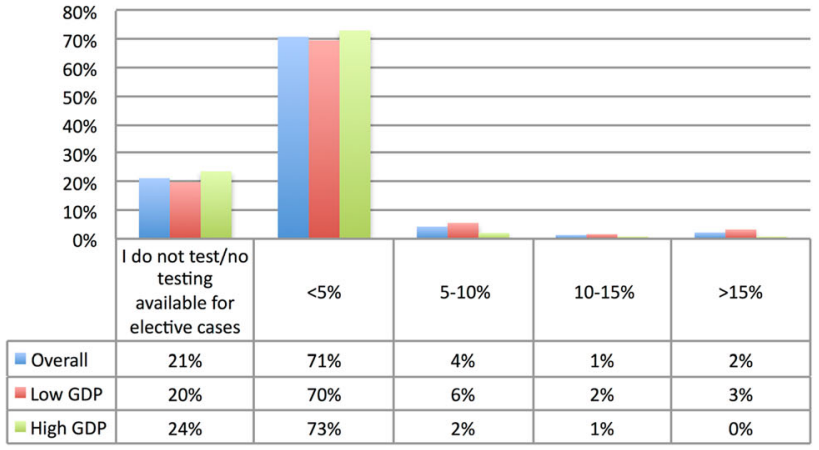

Figure 1a-9a Comparison of high and low gross domestic product per capita (GDP p.c.) countries. Countries were divided into countries with high GDP per capita (TOP30 countries; here referred to as "high GDP”; $n=251)$ and countries with low GDP per capita (>30 countries; here referred as "low GDP"; $n=440$ ), and then, these groups were set in relation to the answers of each question. An overall group $(=$ Overall $(n=691))$ served as a reference bar. Data are demonstrated as percentages (\%). Source for GDP per capita: World
2A

How would YOU estimate patient traffic and demand for aesthetic surgical procedures since resuming activity?

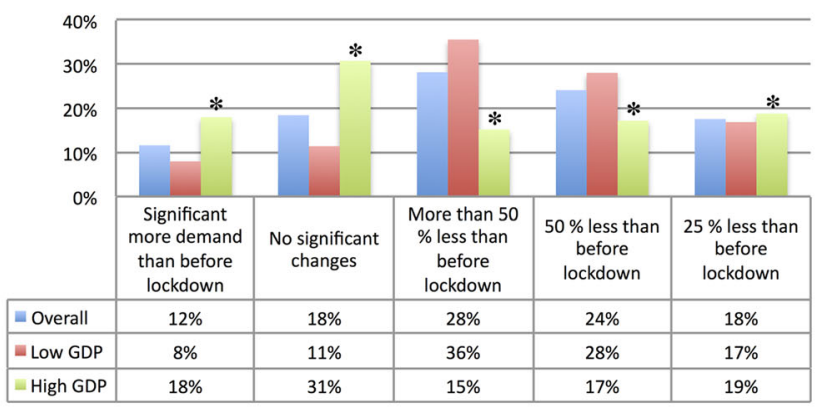

4A

How did YOU implement the ISAPS recommendations regarding presurgical testing in elective aesthetic procedures?

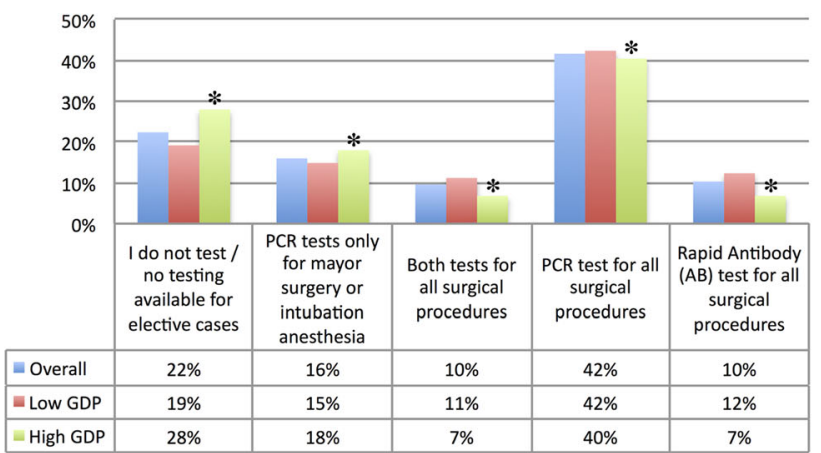

6A

How high is the percentage of complications that may be related to COVID-19 infection (either passed before or newly acquired within the perioperative period) in YOUR surgical patient cohort since resuming elective activity?

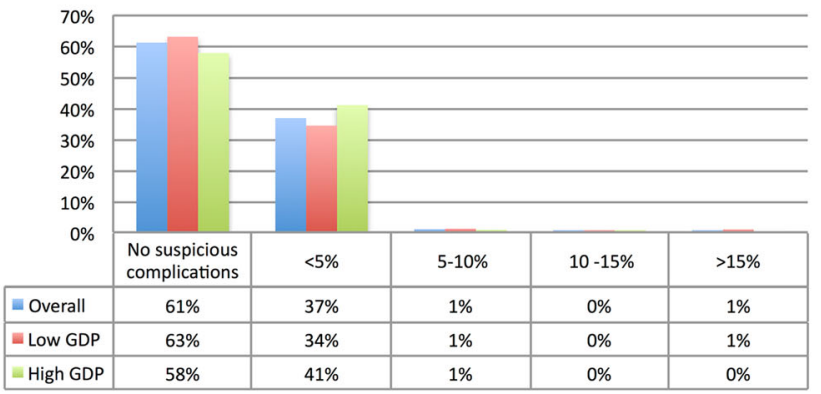

Bank [7]. * = statistically significant results based on Chi-square test results and $r_{\mathrm{s}}$ coefficients: Q1: high GDP $(r=0.8) / \mathrm{Q} 2$ : high and low GDP $(r=-0.7) / \mathrm{Q} 3$ : high and low GDP $(r=-0,6) / \mathrm{Q} 4: \mathrm{H} 0(r=1) /$ Q6: high and low GDP $(r=0.1) / \mathrm{Q}$ : high and low GDP $(r=0.9) / \mathrm{Q} 8$ : high and low GDP $(r=0.8) / \mathrm{Q} 9$ : high and low GDP $(r=0.8) / \mathrm{Q} 10$ : high GDP $(r=0.6)$; Q11: H0 $(r=1) /$ Q12: high GDP $(r=1) /$ Q16: H0 $(r=0.9) / \mathrm{Q} 17: \mathrm{H} 0(r=1) / \mathrm{Q} 18:$ high and low GDP $(r=1)$. 
7A

How many of the ISAPS recommendations regarding adaption of YOUR clinical structure/patient flow did YOU implement (glass shield (reception), hand sanitizer stations, patient guidance system, modification of waiting room, larger spacing of appointments)?

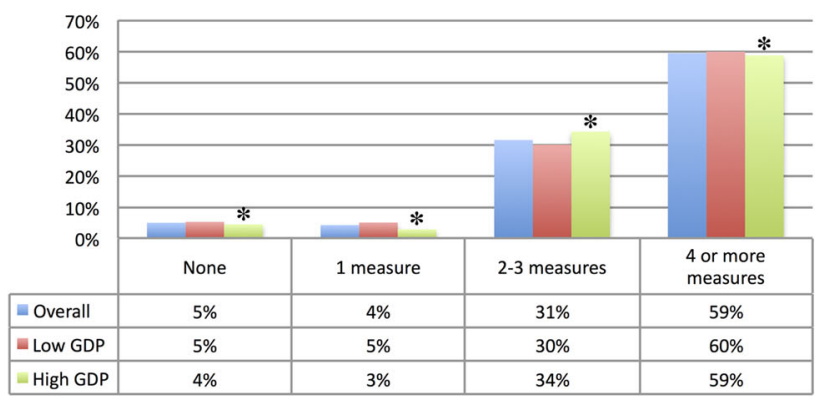

\section{A}

Do YOU consider maintaining some of the ISAPS recommendations/ adaptations even after the pandemic will not been considered a current threat anymore in YOUR country/region because YOU find them useful for patient safety, hygiene, staff safety etc.?

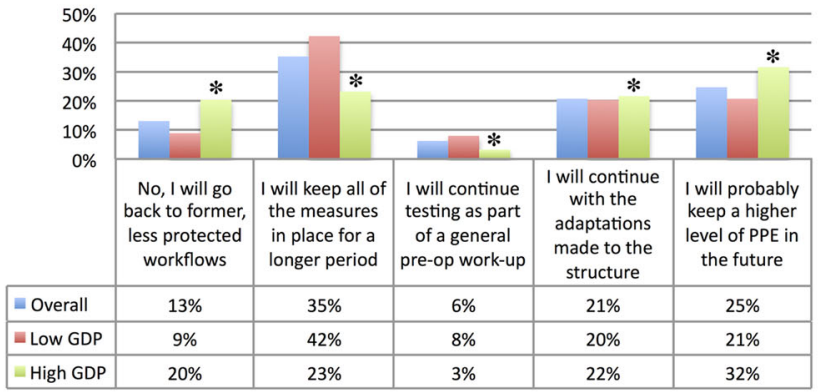

Figure 1a-9a continued

screening, with $49 \%$ of the participants even reporting implementation of all measures. No significant difference could be seen in relation to GDP p.c. or world region.

\section{Question 5-Most Important ISAPS Recommendation for (Pre-) Appointment Screening (See Online Material; Fig. II A/B)}

Wearing a mask is reported to be the most commonly used protective measure worldwide, with Africa + ME participants emphasising this measure more than other regions (overall group: $45 \%$; Africa + ME: 52\%).

\section{Question 6-8-Kind of PPE Used by Clinical Staff (See Online Material; Fig. III-V A/B)}

The use of PPE during consultations (see online material; Fig. III A/B), close examinations, dressing changes or nonsurgical, minimal invasive treatments (see online material; Fig. IV A/B) and surgical interventions (see online material; Fig. V A/B) shows significant differences in the GDP
8A

Which one of the ISAPS recommendations regarding adaption of YOUR clinical structure/patient flow is the most important in YOUR opinion?

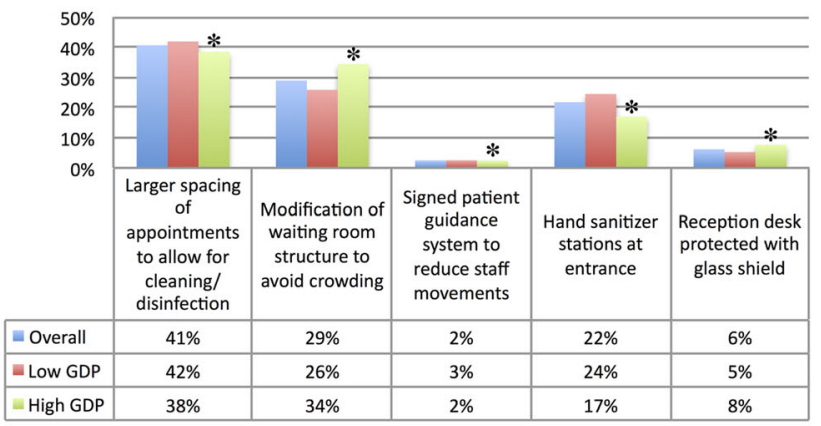

subgroups with more countries from the high GDP p.c. group reporting a use of basic-level PPE (e.g. only surgical masks) and significantly more countries from the low GDP p.c. group reporting a use of advanced-level PPE (N-95 respirator mask / FFP2/3, combination with Face shield etc.).

This is an interesting fact and contradicts the common presumption that more cost-intensive, advanced-level PPE would be more easily available or affordable in countries with high GDP p.c.. Reasons for this could be a different perception of effectiveness of PPE or a lower availability and/or relatively higher acquisition price due to absorption of a larger amount of available advanced-level PPE through the public healthcare systems in the high GDP p.c. group.

Comparing the regional subgroups with the overall group for PPE usage during surgical interventions, Europe reports a relative higher usage of basic-level PPE (surgical masks and single gloves only; overall group: 34\%; Europe: $50 \%$ ). These data could reflect the situation that in many countries in Europe advanced-level PPE equipment was requisitioned by their national public healthcare systemsmeaning that private surgery providers had less access. 


\section{Question 9-Selective Use of PPE (See Online Material; Fig. VI A/B)}

The high GDP p.c. subgroup made their decision for use of basic-level or advanced-level PPE mostly dependent on the type of surgery, which was carried out, and estimated exposure risk (statistic significant), while in the low GDP p.c. subgroup $30 \%$ (vs. $15 \%$ in the high GDP p.c. group) reported to use advanced-level PPE in all cases (see online material; Fig. VI A). An additional exposure risk through aerosols in procedures of the airways and respiratory mucosa has been described, but to the authors' knowledge there are no published data on the difference in exposure risk, especially in operating rooms equipped with a HEPA air filtration system [10]. On the other hand, most consultations and minimal-invasive procedures such as filler and botulinum toxin injections last minimum 15 minutes and are performed in small treatment rooms or offices without air filters. Furthermore, the patient may not be able to wear a mask due to target injection sites in facial treatment. So a differentiation between an expected high or low exposure risk depends on various factors, and a thorough evaluation of each treatment or surgery setting is paramount in the authors' opinion-making the use of advanced-level PPE during certain types of non-invasive treatments more recommendable than in some surgical procedures. The authors expect that with more data on the actual exposure risk is becoming available, official recommendations will most likely change in the future, and the use of advanced-level PPE such as FFP2, FFP3, KN95 or N95-masks for certain non-surgical treatments will become more common in the daily clinical practice, especially when market prices drop as shortage of supply is expected to become less frequent. Until a vaccine becomes available for the general public, the risk for acquiring and spreading a potentially lifethreatening disease with possible long-term complications should be minimized, especially in the reputational context of providing non-essential, elective aesthetic services.

\section{Question 10-Staff's PPE Usage During Beauty Treatments (See Online Material; Fig. VII A/B)}

The trend, which survey participants from high GDP p.c. countries report to use more basic-level PPE for consultations and minimal invasive treatments, continues as well for non-invasive spa and beauty treatments performed by the clinic staff. The low GDP p.c. subgroup reports use of advanced-level PPE (e.g. N-95 respirator masks) for these tasks. When comparing the different world regions, Europe and SA are the regions using a more basic-level PPE for these tasks (see online material; Fig. VII B).

\section{Question 11-Pre-Surgical Testing (Fig. 4 A/B)}

While PCR testing for all surgical procedures was the most reported (42\%) method of preoperative screening, independent from GDP p.c. group or region. (Fig. 4a), high GDP p.c. countries reported at a significantly higher rate for not testing or to not having testing available for elective procedures than compared to the low GDP p.c. group ( $28 \%$ vs. $19 \%$, Fig. 4a). Absorption of laboratory testing capacity through extensive testing by the public sector could be one explanation for this effect. Another reason could be that aesthetic service providers may outsource these additional costs through public testing programs wherever available. When comparing the different world regions, Africa + ME (36\%), Asia + OC (34\%), and SA (29\%) performed less testing or did not have testing available when comparing them to the overall group (22\%) (Fig. 4b).

\section{Question 12-Average Cost for PCR Test (See Online Material; Fig. VIII A/B)}

At time of this survey, the average price for PCR testing was mostly reported to range between $\$ 50$ and $\$ 100$ (see online material; Fig. VIII A/B). As PCR testing involves labor, equipment and physical space, the laboratory costs generally are in correlation to general production costs and level of income in of each country. In countries with higher living costs such as Europe and NA, more than 40\% reported average costs between $\$ 100$ and $\$ 200$ or more (see online material; Fig. VIII B).

\section{Question 13-Average Cost for Antibody Test (See Online Material; Fig. IX A/B)}

At time of the survey, the average price for antibody (AB) testing was mostly reported to be below $\$ 50$. AB testing was reported be cheaper in low GDP p.c. countries (see online material; Fig. IX A). In respect to the data from world regions, prices for $\mathrm{AB}$ testing in Asia $+\mathrm{OC}$ were reported to be up to $46 \%$ cheaper than $\$ 50$ (see online material; Fig. IX B). In comparison with PCR testing, pricing for ready-to-use $\mathrm{AB}$ test is expected to be more independent from general production costs in a country as there are almost no labor or infrastructure cost involved, while distribution and transport cost become more relevant. This may be the reason for the reported differences since for the most test arrays are manufactured in Asia. In the data from the regional groups, $59 \%$ of the participants from Africa reported that no testing was performed or available 
$1 B$

If you stopped practicing, how many weeks after pandemic peek in YOUR country/ region did YOU resume elective aesthetic surgery procedures?

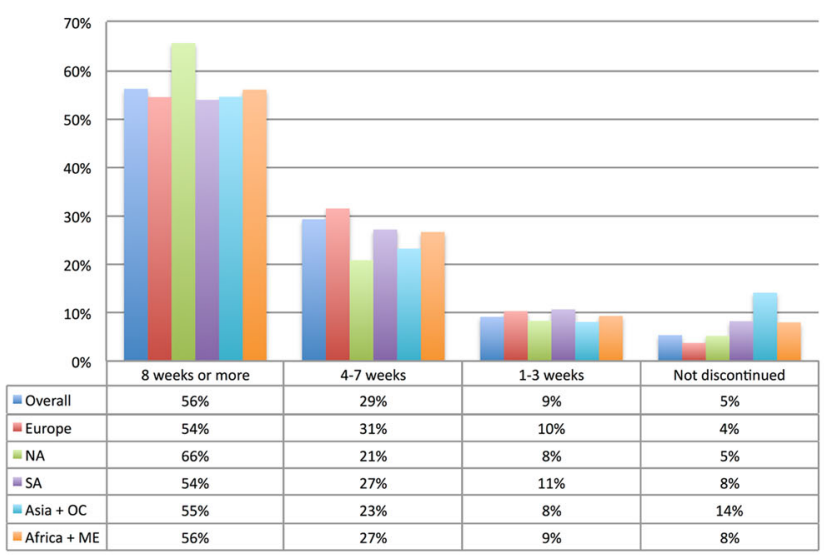

\section{B}

How would YOU estimate patient traffic and demand for aesthetic non-surgical procedures since resuming activity?

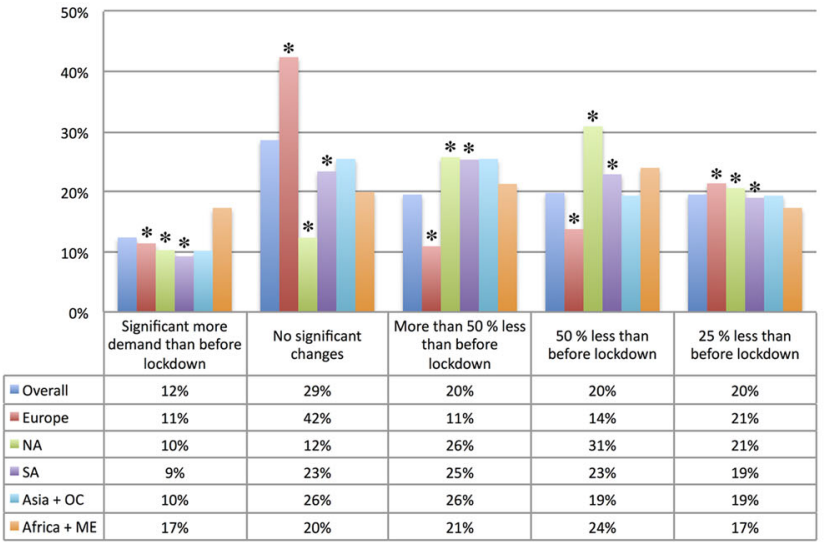

\section{B}

How high is the percentage of positive tests ( $\mathrm{PCR}$ or rapid $\mathrm{AB}$ ) within YOUR patient cohort since resuming elective activity?

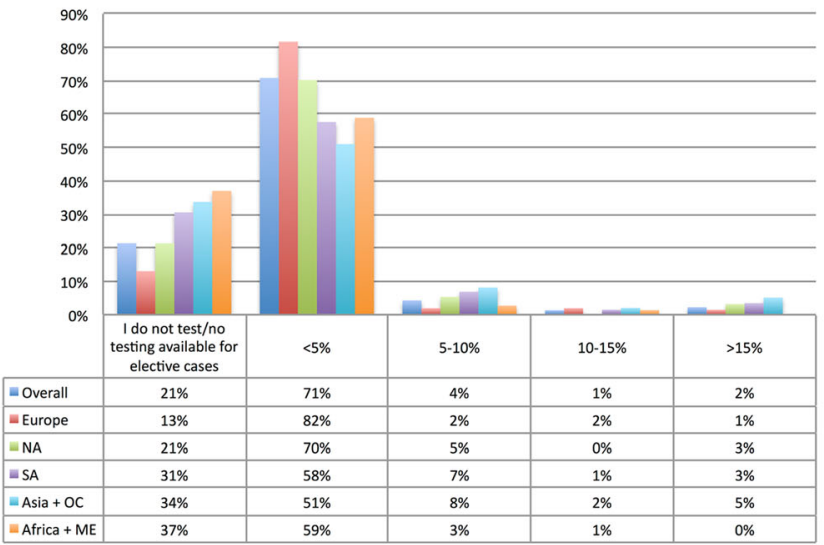

2B

How would YOU estimate patient traffic and demand for aesthetic surgical procedures since resuming activity?

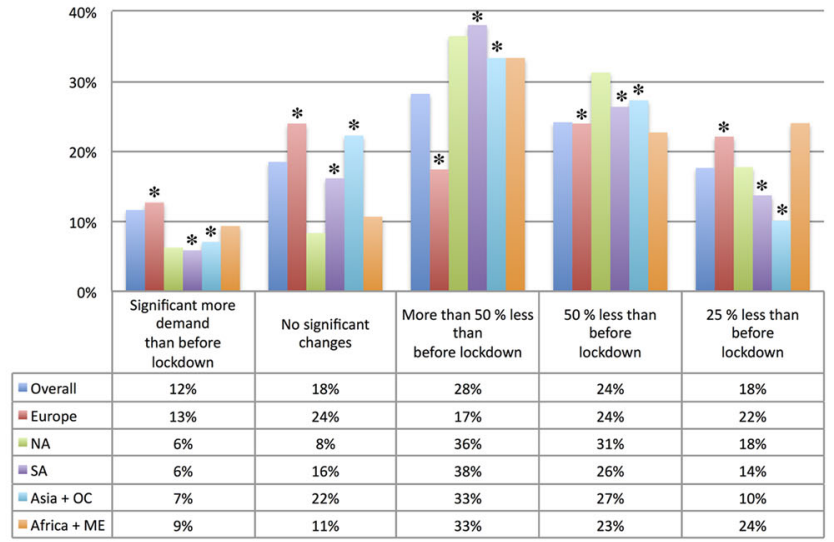

$4 B$

How did YOU implement the ISAPS recommendations regarding pre-surgical testing in ective aesthetic procedures?

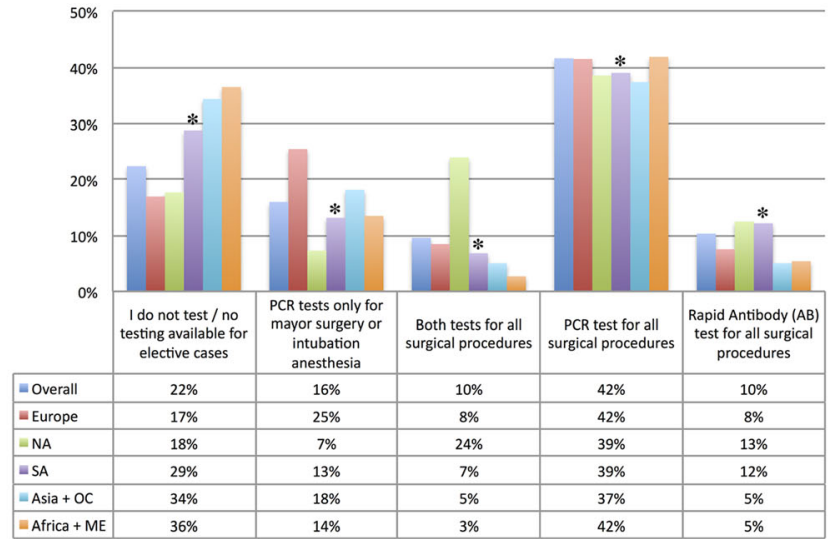

6B

How high is the percentage of complications that may be related to COVID-19 infection (either passed before or newly acquired within the perioperative period) in YOUR surgical patient cohort since resuming elective activity?

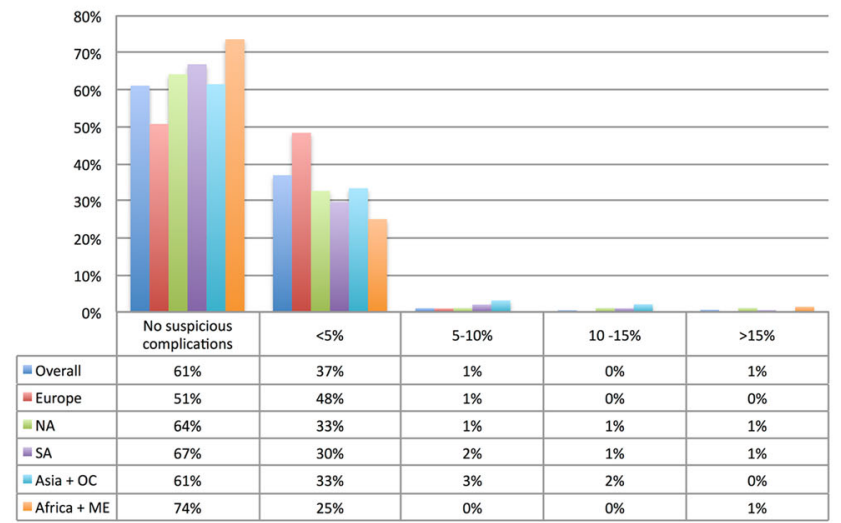


4Figure 1b-9b Comparison of five different world regions. The world was divided into five world regions (Europe $(\mathrm{n}=214)$; North America (NA; $n=97$ ); South America (SA; $\mathrm{n}=206$ ); Asia and Oceania (Asia + OC; $n=99$ ); Africa and Middle East (Africa + ME; $n=75$ ), and then, these regions were set in relation to the answers of each question. Definition about which country belongs to which world region are given in the Material and Method section of this publication. An overall-group (= Overall $(n=691)$ ) served as a reference bar. Data are demonstrated as percentages $(\%){ }^{*}=$ significant statistical results based on $\chi^{2}$ - test results and $r_{\mathrm{s}}$ coefficients: Q2: Europe $(r=-0.6)$; SA $(\mathrm{r}=0.9)$; Asia + OC $(r=$ $0.97) / \mathrm{Q} 3$ : Europe $(r=0.36)$; NA $(r=0.13)$; SA $(r=0.36)$; Asia + OC $(r=0.46)$ / Q6: Europe $(r=0.67)$; SA $(r=0.6)$; Asia + OC $(r=0.3)$ / Q9: Europe $(r=0.8)$; NA $(r=0.01)$; SA $(r=0.8)$; Asia + OC $(r=0.8)$ / Q10: Europe $(r=0.9)$; SA $(r=0.7) / \mathrm{Q} 11: \mathrm{SA}(r=0.6) / \mathrm{Q} 12: \mathrm{NA}$ $(r=0.8)$; Africa $+\mathrm{ME}(r=0.6)$; SA $(r=0.7)$.

for elective cases (see online material; Fig. IX B). That could be an indicator for an undersupply of this world region at the time of our survey.

7B

How many of the ISAPS recommendations regarding adaption of YOUR clinical structure/patient flow did YOU implement (glass shield (reception), hand sanitizer stations, patient guidance system, modification of waiting room, larger spacing of appointments)?

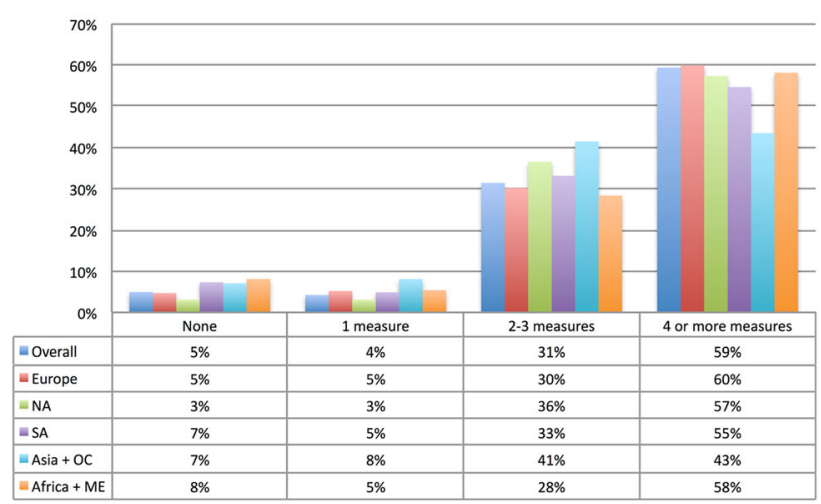

9B

Do YOU consider maintaining some of the ISAPS recommendations/adaptations even after the pandemic will not been considered a current threat anymore in YOUR country/region because YOU find them useful for patient safety, hygiene, staff safety etc.?

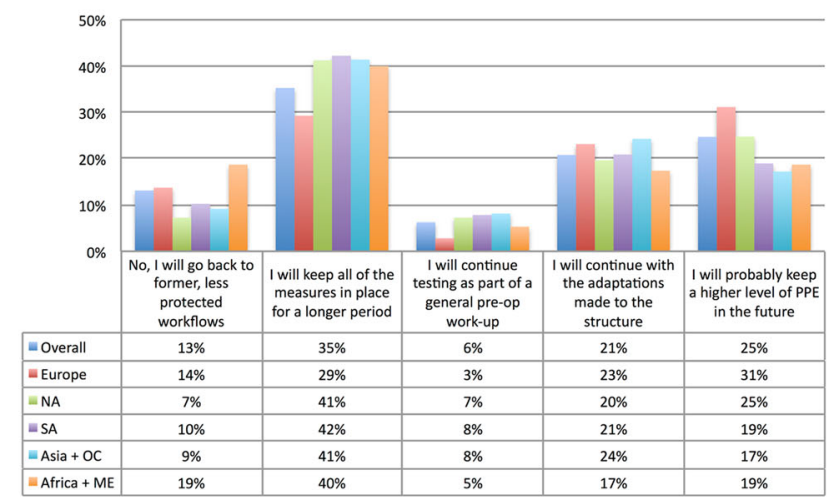

Question 14-COVID-19+ PCR or Antibody Test Results (Fig. 5 A/B)

Independent from GDP p.c. groups or regional subgroups, the majority of survey participants reported less than $5 \%$ of COVID19+ results by PCR or AB testing (Fig. 5 A/B). For a correct interpretation, this relative low incidence rate should be seen in context with the current levels of pandemic infection at time of survey in each country [1]. In most countries, elective surgery was resumed after complete flattening of the first pandemic wave, so a relative low community prevalence is to be expected to reflect in a low positive test rate. With many countries now experiencing a second wave with even higher community case numbers than in the first wave, but without community lockdown or stop of elective surgery, the incidence rate is expected to raise; therefore, the authors recommend to maintain or intensify testing for elective aesthetic procedures rather than stopping testing. General testing of all patients before performing minimal-invasive treatments will remain

\section{B}

Which one of the ISAPS recommendations regarding adaption of YOUR clinical structure/patient flow is the most important in YOUR opinion?

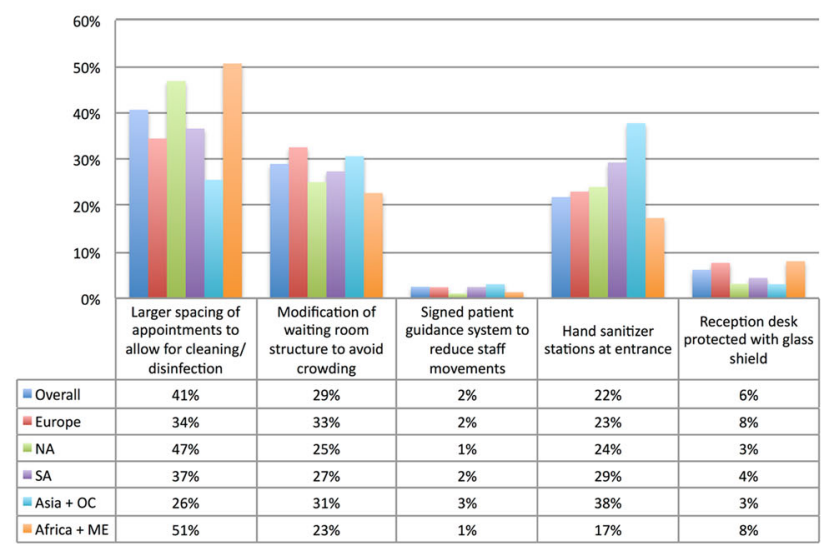

Figure1b-9b continued 
impractical for most aesthetic providers due to the high additional costs in relation to the treatment price. In addition, the rapid $\mathrm{AB}$ test arrays, which are relatively cheap and quickly to perform in the office setting, are not able to detect a recent infection. Rapid SARS-CoV2 antigen tests became recently available and may offer a cost and time effective solution for intensified in-office testing, but the low specificity and higher rate of false-negative results compared with PCR testing remains an issue [11].

\section{Question 15-COVID-19 Related Complications} (Fig. 6 A/B):

Ninety-eight per cent of the survey participants did either not observe any COVID-19-associated perioperative complications $(61 \%)$ or reported a rate $<5 \%$. While it remains paramount to inform the patient about the possibility of an increased perioperative risk while undergoing elective surgery during the COVID-19 pandemic, these observations allow a better and more differentiated analysis of the actual existing risks for patients undergoing elective surgery during a pandemic but are submitted to strict preoperative screening and testing. The results from this survey represent a far larger data set than the data sets used in earlier, monocentric publications that suggested a relatively high perioperative risk [1]. Therefore in the authors' opinion, the actual, additional perioperative risk is well below $5 \%$.

\section{Question 16-Amount of implemented ISAPS recommendations for clinical structure/patient flow (Fig. 7 A/B)}

In the overall group, $90 \%$ of survey participants reported implementation of " 2 or more measures" adapting clinical structure/patient flow to COVID-19 requirements, with "4 or more measures" being the most selected answer (59\%). This answer distribution was homogenous with no significant differences for the GDP p.c. subgroups or the regional subgroups (Fig. 7A/B). The high global implementation rate suggests that most aesthetic service providers agree with the ISAPS guidelines issued on this subject and adopted them widely into their daily clinical practice.

\section{Question 17-Most Important ISAPS Recommendation for Clinical Structure/Patient Flow (Fig. 8 A/B)}

In the overall group, $41 \%$ of the participants considered "more time between patient appointments to allow for cleaning and disinfection" as the most important measure, while "modification of the waiting room structure to avoid crowding" (29\%), and "hand sanitizer stations at the entrance" (22\%) were considered less important (Fig. 8 $\mathrm{A} / \mathrm{B})$. While there was no significant difference in the answer distribution between the GDP p.c. subgroups and the regional subgroups, there seems to be a trend for Asia + OC to rely more on hand disinfection than on patient spacing or room disinfection (Fig. 8b).

\section{Question 18-Maintaining ISAPS Recommendations After End of Pandemic (Fig. 9 A/B)}

The majority of survey participants considers keeping some or all of the newly implemented safety standards for a longer time in the post-pandemic future. Only $13 \%$ of the overall group considered shifting back to former, less protected workflows (Fig. 9a), with no statistically significant difference between regional subgroups or GDP p.c. subgroups.

\section{Conclusions}

The data obtained by this survey allow a more differentiated analysis on the impact of COVID-19 on aesthetic service providers during the first year of the pandemic. It shows that the long work interruption in most countries combined with a general insecurity of prospective clients regarding their midterm socio-economic situation during and after the pandemic caused a significant reduction in patient traffic and demand for aesthetic services for around $70 \%$ of the survey participants. Low GDP p.c. countries experienced a bigger negative economic impact (less aesthetic (non-)surgical procedures), whereas the high GDP p.c. subgroup was less affected by the COVID-19 crisis. Comparing NA and SA subgroups individually to the overall group, these two world regions showed significant less patient flow with a connected higher loss in revenue during the summer of 2020 . This situation may lead to a prolonged and severe loss of income with a reduction of patient traffic for the months to come, since a second wave of the pandemic is ongoing and further lockdowns and operating restrictions may be the consequence until a vaccination of most of the world's population will be carried out. On the other hand, there are as well sources that report a high demand for plastic surgery procedures in certain countries.

In regard to COVID-19 testing, it strikes that $28 \%$ of participants from the high GDP p.c. subgroup and $22 \%$ of the overall group report not to test or to not have testing available for elective procedures. This seems a very high 
percentage taking into account the possible operational or reputational risk of having a COVID-19+ patient in a clinical surgery circuit with a prolonged risk for crosstransmission to healthcare workers, which might lead to a temporary closure of the affected facility.

Although in general most of the ISAPS recommendations on pre-appointment screening, modification of clinical workflow and use of PPE have been implemented by the survey participants, significant differences regarding the level of PPE being available during daily clinical work could be observed. For surgical and non-surgical aesthetic procedures, in the high GDP p.c. subgroup basic-level PPE (surgical mask) was used, whereas the low GDP p.c. subgroup relied more on advanced-level PPE (N-95 respirator mask or higher). Comparing the different world regions subgroups, Europe and Africa used more basic-level PPE than the rest.

Additionally, the overall reported level of PPE used for potentially time-intensive, non-invasive procedures like botulinum toxin and filler injections or medical spa procedures (often being performed in smaller offices with lack of ventilation) seems not to be adequate to the real exposure risk in comparison with the PPE used for surgical interventions, where contamination risk seems to be much lower due to pre-procedure testing and presence of HEPA air filtration systems. Since effective PPE for healthcare workers is a key measure in order to reduce cross contamination and to limit intra-communitarian spread, future revisions of guidelines for aesthetic service providers should take into account the findings of this survey and formulate aligned and refined recommendations to unify the PPE usage throughout the worldwide community.

Based on these data, further recommendations can be made for coping strategies and guideline revisions during future waves of the COVID-19 crisis or for future pandemics. Further follow-up surveys should be considered to get an even fuller insight on the long-term impact of this first global pandemic in modern times. Since the COVID19 pandemic is an international crisis, aligned, expedient and universal actions should be taken.

Acknowledgements No financial contribution of any kind has supported this publication. No grants were accepted for this publication.
None of the authors has any personal or institutional financial interest in this submission.

\section{Compliance with ethical standards}

Conflict of interest The authors have no conflict of interest to disclose.

\section{References}

1. Kaye K, Paprottka F, Escudero R et al (2020) Elective, nonurgent procedures and aesthetic surgery in the wake of SARSCOVID-19: considerations regarding safety, feasibility and impact on clinical management. Aesthetic Plast Surg. 44(3):1014-1042

2. Singh RK, Rani M, Bhagavathula AS et al (2020) Prediction of the COVID-19 pandemic for the top 15 affected countries: Advanced Autoregressive Integrated Moving Average (ARIMA) model. JMIR Public Health Surveill. 6(2):e19115

3. Johns Hopkins University (2020) COVID-19 dashboard by the center for systems science and engineering (CSSE) at Johns Hopkins University (JHU). https://coronavirus.jhu.edu/map.html. Accessed 19 Sept 2020

4. Middleton J, Reintjes R, Lopes H (2020) Meat plants-a new front line in the covid-19 pandemic. BMJ 370:m2716

5. Doglietto F, Vezzoli M, Gheza F et al (2020) Factors associated with surgical mortality and complications among patients with and without coronavirus disease 2019 (COVID-19) in Italy. JAMA Surg. 155:691

6. Abdi M (2020) Coronavirus disease 2019 (COVID-19) outbreak in Iran: actions and problems. Infect Control Hosp Epidemiol. 41(6):754-755

7. World_Bank (2020) GDP per capita (current US\$)

8. Fisher RA (1922) On the Interpretation of $\chi 2$ from Contingency Tables, and the Calculation of P. J R Stat Soc 85(1):87-94

9. Lovie AD (1995) Who discovered Spearman's rank correlation? Br J Math Stat Psychol 48:255-269

10. Chisari E, Krueger CA, Barnes CL, Van Onsem S, Walter WL, Parvizi J (2020) Prevention of infection and disruption of the pathogen transfer chain in elective surgery. J Arthroplasty. 35(7S):S28-S31

11. Mak GC, Cheng PK, Lau SS et al (2020) Evaluation of rapid antigen test for detection of SARS-CoV-2 virus. J Clin Virol. 129:104500

12. Meeson S (2020) Why plastic-surgery demand is booming amid lockdown. BBC

Publisher's Note Springer Nature remains neutral with regard to jurisdictional claims in published maps and institutional affiliations. 